\title{
Balanced automatization levels in manufacturing systems
}

\author{
A. Adlemo \\ Dept. of Computer Engineering, Chalmers University of Technology \\ S-412 96 Göteborg, Sweden \\ tel. +46-31-772 1000, fax. +46-31-772 3663 \\ adlemo@ce.chalmers.se
}

\author{
S.-A. Andréasson \\ Dept. of Computing Science, Chalmers University of Technology \\ S-412 96 Göteborg, Sweden \\ tel. +46-31-772 1043, fax. +46-31-16 5655 \\ andreasson@cs.chalmers.se
}

\begin{abstract}
The role of the human operator in manufacturing systems has changed in recent decades as computers, data networks and intelligent machines have been introduced. One effect is that the number of operators has been reduced as the cost of operators is higher than the cost of computers, intelligent machines and intelligent transportation systems. However, to reach a balanced automation system, it is necessary to consider the relative cost of labor as compared with advanced machinery. At the same time, it is essential that these considerations do not affect product quality.

The way of reaching a balanced automation system presented in this paper is through appropriate levels of control and automatization, considering cost and product quality. It is demonstrated how a modern machining cell can be stripped, according to the described levels of automatization, to save money and thus reach a balanced automation system without any loss of product quality. The bottom line of the discussion is that product supervision should not be left to human operators solely, but rather be performed automatically by the system. Apart from the machining cell, an assembly cell in a car manufacturing installation is also demonstrated to explain the full range of aspects of automatization when designing a balanced manufacturing system.
\end{abstract}

\section{Keywords}

Balanced automation systems, computer integrated manufacturing, quality control 


\section{INTRODUCTION}

The role of the operator in a manufacturing system has changed with time. In early days of manufacturing, the tasks of an operator were to manually load, operate and unload machines. Later on, more advanced machines were introduced that could work autonomously, although without intercommunication. As progress continued towards more intelligent machines and with the introduction of computers, the manufacturing system was expected to take over more and more of the operator's work and finally become the ultimate, unmanned factory.This view has been moderated slightly in recent years and the role of the operator in a manufacturing system has become more appreciated. The knowledge and experience of a skilled operator is very difficult and expensive to achieve and build into a system in other ways.

Various papers have been written on the role of the human operator in manufacturing systems, e.g:

- Operator control activities, Adlemo and Andréasson (1995a), Sheridan et al. (1988).

- Operator roles and conflicts, Badham and Schallock (1991), Drury et al. (1986), Mårtensson and Stahre (1992), Stahre and Mårtensson (1992).

- Operator interfaces, Geary et al. (1992), Olsson and Lee (1994), Schneiderman (1987), Sylla (1992).

The tendency in modern manufacturing systems has been to reduce human interactions with systems to a minimum. One important reason for this, albeit not the only one, has been to reduce costs caused by increases in the salaries of human operators. At the same time, the cost of introducing intelligent machines and automatic control systems has decreased in relative terms as compared with operators' salaries. Reductions in human interactions with a manufacturing system, and thus indirect reduction in the number of human operators involved in production, are understandable adjustments made by chief executives. Humans are notoriously inconsistent, both inter-individually and over time. Humans tire easily. They often reject products they regard with suspicion more on the basis of fixed quotas than on actual defect levels. Computers and machines, on the other hand, are very patient supervisors, over long periods of time, and neither complains nor become bored. An important aspect, however, is how to obtain a balanced manufacturing system, meaning a system that has the proper mix of operators and machines to obtain the highest profit possible, taking into account the country in which the system is located, and without suffering any losses of product quality.

This paper discusses the levels of control and automatization in a manufacturing system appropriate to obtain a balanced system. This is achieved by studying a system and identifying conditions under which it is best to rely on an operator or on an automatic system. This adjustment is made considering various aspects, such as time (i.e. productivity), money (i.e. savings), safety (i.e. human safety), flexibility and quality. The objective of the research presented in this paper is to demonstrate situations in which an operator can perform a work task rather than have the system do it automatically. To illustrate this, a machining cell for the production of truck and bus axles is used. The machining cell is stripped in a number of steps, from a totally automatic system to a totally manual system. The main theme of the discussions in this paper is that, even when human operators perform activities in a manufacturing system that would normally be considered appropriate for automatic control and automatic production, the supervisory portion of the control should not be left to the operators solely if the quality goals of production are to be met. One reason for this is that the equipment needed for 
supervision (computers, data network and sensors) is relatively cheap as compared with advanced machines, such as a computerized milling machine. To obtain $100 \%$ inspection using humans typically requires a considerable amount of redundancy, often as much as three reinspections (Dreyfuss, 1989; Freeman, 1988). At the end of the paper is given another example of a balanced manufacturing system. Here, however, an assembly cell in a car manufacturing installation is used rather than a machining cell.

The work presented in this paper is the result of work by a cross departmental research group at Chalmers University of Technology, Göteborg, Sweden. The group consists of individuals from the Department of Computer Engineering, Department of Computing Science, Department of Production Engineering and the Control Engineering Laboratory. Other work performed by the group relates to high level operational lists (Andréasson et al., 1995), the control system in a machining cell (Fabian et al., 1995) and generic resource models (Gullander et al., 1995). The research aims at demonstrating how a truly flexible manufacturing system can be achieved (see Adlemo et al., 1995b, for some preliminary results).

\section{CASE STUDY OF A MACHINING CELL}

A machining cell for rear-axles has recently been installed at one site at Saab Scania Trucks and Buses in Sweden. The cell serves as an example of a highly automatic manufacturing system and of how a balanced system can be achieved in a country in which salaries are comparatively high. However, the following sections demonstrate how this system can be stripped of different parts to obtain balance in a country in which salaries are comparatively low.

As can be observed in Figure 1, the cell consists of seven resources; a lathe and a multi operational milling device, together with a quality control (QC) station; two exit buffers and one entry buffer; and a gantry crane (GC) for loading and unloading the devices. A local area network interconnects the resources with each other and with the cell controller. An initial premise was that all communication should take place by means of MMS messages (MMS, 1990). Finally, however, only the Read- and WriteVariable messages were chosen to be implemented.

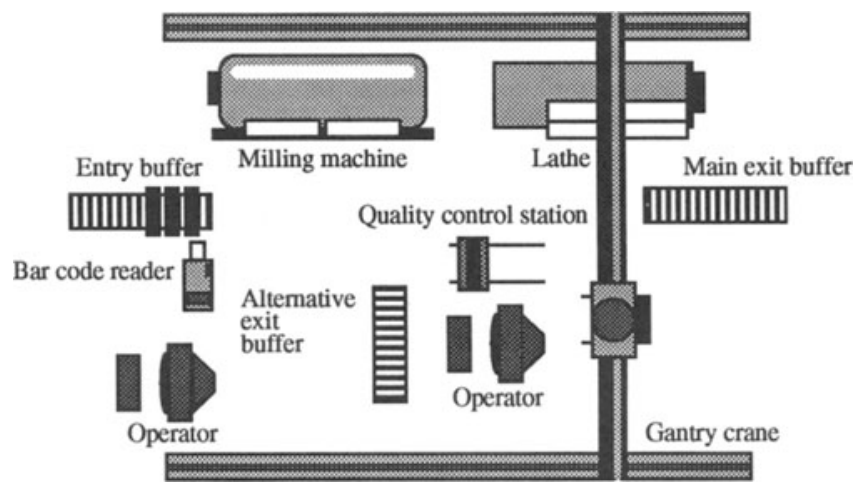

Figure 1 The machining cell in the case study. 
Rear axles are manually entered by the operator at the entry buffer. Here, the bar code reader registers the incoming axles by identifying their article number. The operator can manually enter rework codes for those axles that have already been through the system but have been rejected by the QC. The normal flow through the system for each axle is first to visit the milling machine and then the lathe, and finally to exit through the main exit. However, the operator (or operators) can at any time request a specific axle to the QC station, where it will be tested for adherence to the tolerance specifications. This testing is done manually.

The GC is a special type equipped with twin grippers. Under normal operation, one of the grippers is always empty, while the other holds an axle. Loading a device with a new axle means first fetching the old axle with the empty gripper, rotating 180 degrees and then loading the device with the new axle. Thus, loading a device is actually an unload/load sequence under normal operation. When leaving an axle at one of the exit buffers, the GC becomes empty, and then always moves to the entry buffer to fetch a new axle. Special work cycles must naturally exist for the starting up and emptying of the cell. The fact that the GC has twin grippers, with one always empty, is equivalent to having one global buffer place within the system. Thus, the system can never deadlock. This fact significantly simplifies the implementation of the control functions.

With production times of about 10 minutes for each device, normal operation means that the GC will spend most of its time waiting for the axles to finish their work cycles. When the GC has fetched a new axle from the entry buffer, it will wait for the milling machine to finish its current task. When the milling machine has been served, the GC stands idle waiting for the lathe to finish its current job. Finally, when the lathe has been unloaded/loaded, the processed axle will be left at the main exit, whereafter the GC fetches a new axle from the entry buffer.

The result of normal operation is that incoming axles push the outgoing material in front of them. Axles will only be unloaded when new axles are to be loaded. This has several important implications. For instance, if the input flow of axles stops, axles will remain in the devices until a new axle forces the other axles to exit the devices. When the system is not in the start-up or emptying phase, there will usually be an axle at the QC station waiting to be moved to some other unit, so that axles may have to wait a good deal of time to be moved from the QC station.

\section{CONTROL AND AUTOMATIZATION CONCEPTS}

The production in the machining cell described in section 2 is designed to be more or less automatic, i.e. human interference in production should be minimum. The only interference is the operator supervising the system. However, the machining cell, as it is presented in the case study, can be stripped of parts of the automatic resources, e.g. the gantry crane. The result of a totally stripped system is a completely manual system with manually operated machines and manual transportations. Where the exact amount of automatization should be placed is governed by a number of concepts, e.g. time, money, safety, flexibility and quality. These concepts and their effect when making decisions are described below. 


\subsection{Time}

The first concept, time, is normally associated with the level of automatization such that greater automatization leads to faster production and thus a reduction in the time spent. However, in some special situations, this may not be true. Another aspect is that it is difficult to manually optimize production, which leads to a longer overall production time.

\subsection{Money}

In second concept, money, it is not as easy to identify the relation between the degree of automatization and the costs or savings. Normally, the replacement of an operator with an automatic equivalent renders some reductions in cost, as the operator is one of the more expensive parts. However, in some countries, the cost of an operator is a relatively small expenditure. This leads to a situation in which it might be more favorable to use humans instead of unmanned, automatic machines.

\subsection{Safety}

When it comes to safety, the advantages of an automatic system are more obvious. The removal of humans in a manufacturing system is almost always a way to reduce the risk of injuries during normal production. However, in terms of service, it is usually safer for an operator to work in a manually operated system.

\subsection{Flexibility}

The advantages of an automatic system over a human operator as concerns flexibility is not as tangible. When it comes to flexibility, who can beat man? For instance, if the space encountered in a manufacturing cell is very limited, it might be more flexible to rely on a human rather than a big, bulky robot.

\subsection{Quality}

The upholding or improvement of production quality, finally, is more directly correlated to the automatization of production. By introducing computers, data networks and intelligent machines into a manufacturing system, quality can be increased. As mentioned initially, humans tire easily and are notoriously inconsistent.

\section{STEPS OF AUTOMATIZATION IN A MANUFACTURING SYSTEM}

The following section describes a number of possible steps of automatic production in a manufacturing system, using the manufacturing cell in section 2 as an example.

Six different steps of automatization have been identified: 
1. Cell control system.

2. Automatic asynchronous material transportation.

3. Automatic synchronous material transportation.

4. Automatically operated machines.

5. Automatic supervision.

6. Data network.

These six different steps of automatization can be combined in a number of possible ways into what we call levels of automatization.

A system that is automatized with respect to cell control system, material transportation, machine operation, data transportation and control supervision represents a fully automated system. The following subsections describe possible ways of reducing the amount of automatization.

\subsection{Cell control system}

One way to reduce the cost of a fully automatized system is to remove the cell control system. As the cell control system takes care of such things as scheduling and dispatching, this means that the worker who takes the control systems place must be highly skilled and, thus, the control system cannot be replaced with any worker.

In the case study, this step of automatization can be obtained by removing the cell controller.

\subsection{Automatic asynchronous material transportation}

The transportation of material between different machines in a machining cell is asynchronous, i.e. there are no requirements concerning synchronization with other flows of transportation.

Another way to reduce the cost of a fully automatized system, apart from the cell control system mentioned above, is to remove the automatic asynchronous material transportation system. Instead, one relies on a manually operated material transportation system between different machines. One hazard when removing the automatic material transportation system is the increased risk of errors introduced by humans. Another hazard is the risk of human accidents when the automatic material transportation system is omitted.

In the case study, this step of automatization can be obtained by replacing the expensive gantry crane with a manually operated truck. Instead, here, printed truck orders are needed. If the quality of the products is to be maintained in this case, it is necessary to use sensors at the machines to verify that the correct product is delivered at the correct machine.

\subsection{Automatic synchronous material transportation}

In an assembly cell, as compared with a pure machining cell, there is also a synchronous transportation of material between different stations. The synchronous transportation is special, as the arrival of a piece of material at a station should be coordinated with the arrival of other pieces of material. This implies that synchronous transportation needs a better, and possibly 
more expensive, supervision than asynchronous transportation. By replacing an automatic synchronous transportation system with a manual equivalent, it is possible to reduce costs. As in asynchronous material transportation, the risk of errors introduced by humans increases.

Another aspect of the synchronous material transportation is that it is sometimes used to hold a piece of material in position while some other parts are joined to it. This type of work is usually performed by a robot that has the capability for both material transportation and material fixation. If synchronous material transportation that should perform both tasks is necessary, it is more difficult to replace this by a worker.

Thus, as synchronous material transportation is sometimes more complicated than asynchronous material transportation, it is advisable to try to omit asynchronous material transportation sooner than any synchronous material transportation.

\subsection{Automatically operated machines}

Another method for reducing costs is to exchange automatically operated machines with semiautomatic or manually operated machines. This can be done for all of the machines or only some of them.

In the case study, this step in reducing automatization can be obtained by replacing the expensive milling machine and the expensive lathe by manually operated equivalents. Instead, here, printed working orders for the machines are needed. If the quality of the products is to be maintained at the same time that automatically operated machines are omitted, it is necessary to use sensors that verify the quality of the products.

\subsection{Automatic supervision}

To further reduce costs it is possible to remove automatic production supervision. Hence, as there are no supervisory activities, it is impossible to verify the quality of the products and, in some cases, impossible to validate the correctness of the products. Instead, these activities must be performed by a human, with the increased risk of erroneous measurements. In spite of this, some information can still be passed through the manufacturing system via the data network.

In the case study, this level is obtained if the automatic production supervision activities are removed, e.g. by removing the bar code reader. The sensors that were introduced earlier to verify the delivery of correct products and the quality of the products are also omitted. Manual routines to verify the quality are then needed.

\subsection{Data network}

Yet another step to reduce costs is to remove the data network. This leaves us with a system with stand-alone machines, where the instructions for the machines must be entered manually as no data network exists to transport instructions.

In the case study, this level can be obtained by removing the local area network that interconnects the machines and the cell controller. 


\section{APPROPRIATE LEVELS OF AUTOMATIZATION IN A MANUFACTURING SYSTEM}

The six different steps of automatization can be combined into different levels of automatization. Table I describes some examples of possible levels of automatization by combining the six steps of automatization in different ways. For example, one level is the fully automated production, i.e. level I. Another level is to introduce manual asynchronous material transportation but to allow the rest to be automatic, i.e. level II. Still another level would be to have everything manually controlled and have no data network, i.e. level VI. A level that actually exists in many installations is automatically operated machines and no further automatization, i.e. level III, although this level inflexible as well as unpractical. Furthermore, verification of the quality of the products is very difficult.

Table 1 Steps of automatization combined to achieve different levels of automatization

1. Cell control system

2. Automatic asynchronous material transportation

3. Automatic synchronous material transportation

4. Automatically operated machines

5. Automatic supervision

6. Data network
Y Automatic or present

N Manual or not present

M Mixed automatic and manual

- Not applicable

Steps of automatization

\begin{tabular}{lllllllll} 
No. & Examples of levels of automatization & 1. & 2. & 3 & 4. & 5. & 6. \\
\hline I & Fully automated manufacturing cell & $\mathrm{Y}$ & $\mathrm{Y}$ & $\mathrm{Y}$ & $\mathrm{Y}$ & $\mathrm{Y}$ & $\mathrm{Y}$ \\
II & Manual asynchronous material transportation & $\mathrm{Y}$ & $\mathrm{N}$ & $\mathrm{Y}$ & $\mathrm{Y}$ & $\mathrm{Y}$ & $\mathrm{Y}$ \\
III & Automatically operated machines only & $\mathrm{N}$ & $\mathrm{N}$ & $\mathrm{N}$ & $\mathrm{Y}$ & $\mathrm{N}$ & $\mathrm{N}$ \\
IV & Lowest acceptable level of automatization & $\mathrm{N}$ & $\mathrm{N}$ & $\mathrm{N}$ & $\mathrm{N}$ & $\mathrm{Y}$ & $\mathrm{Y}$ \\
$\mathrm{V}$ & Not acceptable level of automatization & $\mathrm{N}$ & $\mathrm{N}$ & $\mathrm{N}$ & $\mathrm{N}$ & $\mathrm{N}$ & $\mathrm{Y}$ \\
VI $\quad$ Non automatic production & $\mathrm{N}$ & $\mathrm{N}$ & $\mathrm{N}$ & $\mathrm{N}$ & $\mathrm{N}$ & $\mathrm{N}$ \\
VII & The machining cell in the case study (section 2) & $\mathrm{Y}$ & $\mathrm{Y}$ & - & $\mathrm{Y}$ & $\mathrm{M}$ & $\mathrm{Y}$ \\
VIII & The assembly cell in the example (section 6) & $\mathrm{Y}$ & $\mathrm{N}$ & $\mathrm{Y}$ & $\mathrm{Y}$ & $\mathrm{M}$ & $\mathrm{Y}$ \\
\hline
\end{tabular}

The conclusion drawn from observing the different levels of automatization is that the lowest acceptable level of automatization for a manufacturer, without having to consider the country or type of production, is the case in which production supervision is left to the automatic 
system. This would be a system described by level IV. If the automatic supervision is removed, i.e. level $\mathrm{V}$, the quality of the products would be affected.

To achieve a balanced manufacturing system, one should omit expensive automatic parts in favor of manual counterparts if the manual counterparts are cheaper in the long run and if the product quality can be maintained.

Taking into consideration the aspects of safety, quality etc., we believe that the following steps should be taken in the following order to achieve a balanced manufacturing system:

- Omit automatic asynchronous material transportation.

- Omit automatic synchronous material transportation.

- Omit automatically operated machines.

We believe that it is most cost effective to have manual material transportation, as described earlier, and then, if a greater reduction in automatization is required, to continue by omitting manually operated machines. The reason for this is that it is easier to maintain a certain level of product quality using manual transportation than it is to introduce manually operated machines, where there is a greater risk of reduced product quality. The next step toward reducing costs is to remove the automatic supervision of production. However, we strongly believe that this is an unwise step, as the verification of the quality of the products is left to a human inspector who is more liable to commit errors than a correctly installed automatic supervision system. Furthermore, the cost of automatic supervision with computers and sensors is far less expensive than highly complex machines and automatic transporting systems. In order to have automatic supervision, it is necessary to have a data network and, hence, this component should not be omitted in a balanced manufacturing system.

\section{EXAMPLE OF A BALANCED ASSEMBLY CELL}

The example describes a non-traditional assembly cell. Two of the primary differences, as compared with traditional cells, are the number of parallel assembly teams resulting in very long cycle times and the material feeding system, which is a pure kitting system. The cell has been studied extensively and the layout and function of the cell is described in Johansson and Johansson (1990). The cell is also illustrated in Figure 2.

This cell produces kits, which are plastic bags containing small size parts (e.g. screws, nuts, and plugs) to be used in the assembly department of a company. Each kit contains the small parts needed for a specific portion of the assembly of one specific product. There is a large variety of kits (several hundred), each containing between two and 20 different parts, i.e. parts with different part numbers, and between 10 to 40 single parts. A typical value is 8 part numbers corresponding to 25 single parts.

The production of kits is divided into two major activities corresponding to physical areas in the system: automatic counting and packaging (consisting of bagging and printing). Counting is done into sectioned trays, where each tray has 18 sections. Automatic counting is done by four identical vibratory bowl feeders that work in a so called counter-group, $P_{1}$. The feeder can handle only one part number at a time, which means that four part numbers per kit is the maximum number that can be counted at one time. For this reason, many of the kits must pass the counter-group more than once. Bagging is done by a bagging machine, $\mathrm{P}_{21}$. In a special machine, the tray is turned and the kits are placed into a bucket elevator that leads to the bagging 
machine. A printer that to the bagging machine prints the internal part number of the kit on the bag, $\mathrm{P}_{22}$. The quality of the kits with regard to part numbers and quantities of each part number is controlled by a check weigher connected to the bagging machine, $\mathrm{P}_{23}$. The downloading of production information is retrieved from the VAXCluster, $P_{-1}$, via the personal computer, $P_{0}$.

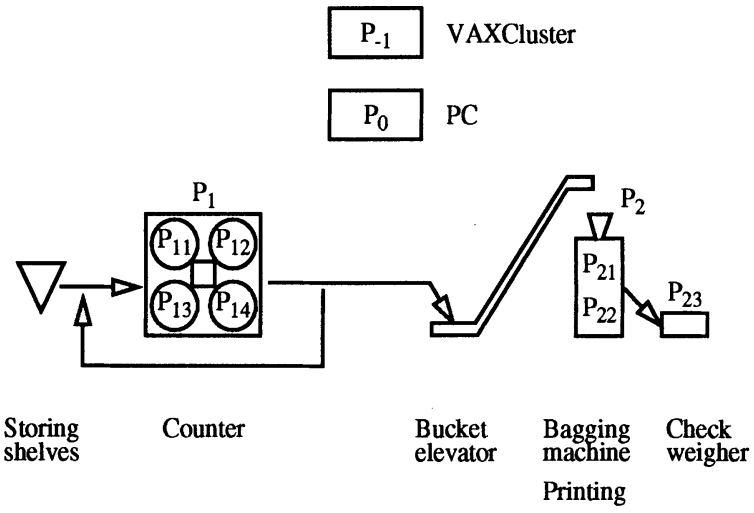

Figure 2 Layout of the assembly cell.

The assembly cell is balanced in the sense that, when the cell was planned, it was decided that the asynchronous material transportation be performed manually, e.g. the transportation between $\mathrm{P}_{1}$ and $\mathrm{P}_{2}$, while the synchronous material transportation be performed automatically, e.g. the positioning of the tray in the $\mathrm{P}_{1}$ area. This measurement was taken in a car producing facility in an industrialized country, i.e. Sweden, even though the salaries were high. The reason for this was simply that the asynchronous material transportation was too expensive to perform automatically. The cell control system is automatic and performed by the personal computer $\mathrm{P}_{0}$. The machines, i.e counting, $P_{1}$, bagging, $P_{21}$, printing, $P_{22}$ and weighing, $P_{23}$, are automatically operated. Most of the supervision is done automatically but an operator manually enters the identification of the tray into the packaging system. Finally, a data network is connected to all the machines in the cell.

The weak part of the assembly cell is the manual supervision. There is an obvious risk for the operator to enter an erroneous identification. We therefore suggest that bar code readers are introduced, both at the counter, $\mathrm{P}_{1}$, and at the packaging system, $\mathrm{P}_{2}$. At $\mathrm{P}_{1}$, the identification of the tray is read and saved in a database where it can be retrieved by $\mathrm{P}_{2}$ afterwards. In this way, the quality of the products is not endangered, as it is the case of manual supervision.

\section{CONCLUSIONS}

A number of levels of automatic control and automatic production have been presented in order to identify activities that may be performed by a human operator rather than an automatic system to achieve a balanced automation system with respect to such relevant aspects as cost, flexibility and quality. To do so it is essential for a manufacturing system that the supervisory portion exists and is performed automatically by the system. It is not possible to leave this task 
to a human operator solely, as it is common for humans to commit errors that might reduce the quality of the products. In addition, the cost of the equipment to perform the automatic supervision is now relatively low, compared with certain highly specialized machines. Nonetheless, certain parts of an automatic system can be omitted to reduce costs without reducing the production quality.

Thus, by removing expensive machines, such as the gantry crane described in section 2 , in exchange for humans and simple, cheap machines, it is possible to reduce costs, maintain the quality of the final product and uphold a higher degree of employment in countries in which salaries are low. However, it is essential in such situations that the manufacturing system be able to rely on some kind of automatic supervision to verify product quality. We believe that the cost of having a reasonable amount of computer power together with a data network that connects the computers with the sensors placed on the machines is reasonable and affordable. Even more important, this equipment is necessary if a manufacturer is to be able to provide products of good quality.

\section{REFERENCES}

Adlemo A. and S.-A. Andréasson (1995a). Operator control activities: a case study of a machining cell. To appear in Proceedings of the 8th IFAC/IFIP/IFORS/IMACS/ISPE Symposium on Information Control Problems in Manufacturing Technology, INCOM'95. Beijing, China.

Adlemo A., S.-A. Andréasson, M. Fabian, P. Gullander and B. Lennartsson (1995b). Towards a truly flexible manufacturing system. Will appear in Control Engineering Practice, 3.

Andréasson S.A., A. Adlemo, P. Gullander, M. Fabian and B. Lennartsson (1995). A machining cell level language for product specification. To appear in Proceedings of the 8th IFAC/IFIP/ IFORS/IMACS/ISPE Symposium on Information Control Problems in Manufacturing Technology, INCOM'95. Beijing, China.

Badham R. and B. Schallock (1991). Human factors in CIM: a human centred perspective from Europe. International Journal of Human Factors in Manufacturing, 1, 121-41.

Dreyfuss D. D. (1989). Is industry ready for machine vision? - a panel discussion, in Machine Vision for Inspection and Measurement (ed. H. Freeman), Academic Press Inc., New York, U.S.A., 223-36.

Drury C. G., M. H. Karwan and D. R. Vanderwarker (1986). Two inspector problem. IEEE Transactions, 14, 174-81.

Fabian M., P. Gullander, B. Lennartsson, S.-A. Andréasson and A. Adlemo (1995). Dynamic products in control of an FMS cell. To appear in Proceedings of the 8th IFAC/IFIP/IFORS/ IMACS/ISPE Symposium on Information Control Problems in Manufacturing Technology, INCOM'95. Beijing, China.

Freeman H. (1988). Machine Vision. Academic Press Inc., Boston, U.S.A.

Geary G. M., R. Walker, H. Mehdi and W. Parks (1992). Improving the yield of a manufacturing process using an on-line product information and display system. Proceedings of the 7th IFAC/IFIP/IFORS/IMACS/ISPE Symposium on Information Control Problems in Manufacturing Technology, INCOM'92. Toronto, Canada, 177-81. 
Gullander P., M. Fabian, S.-A. Andréasson, B. Lennartsson and A. Adlemo (1995). Generic resource models and a message-passing structure in an FMS controller. To appear in Proceedings of the 1995 IEEE International Conference on Robotics and Automation, ICRA'95. Nagoya, Japan.

Johansson M. I. and B. Johansson (1990). High automated kitting system for small parts - a case study from the Volvo Uddevalla plant. Proceedings of the 23rd International Symposium on Automotive Technology and Automation. Vienna, Austria, 1, 75-82.

MMS (1990). Industrial Automation Systems - Manufacturing Message Specification -Part 1: Service Definition, International Standard, ISO/IEC 9506-1, First edition.

Mårtensson L. and J. Stahre (1992). Operator roles in advanced manufacturing, in Ergonomics of Hybrid Automated Systems III (eds. P. Brödner and W. Karwowski), Elsevier, Amsterdam, The Netherlands, 155-62.

Olsson G., P. L. Lee (1994). Effective interfaces for process operators - a prototype. Journal of Process Control, 4, 99-107.

Schneiderman B. (1987). Designing the User-Interface: Strategies for Effective HumanComputer Interaction, Addison-Wesley, Reading, U.S.A.

Sheridan T. B., L. Charny, M. B. Mendel and J. B. Rosenborough (1988). Supervisory control, mental models and decision aids. Proceedings of the 3rd IFAC Conference on Analysis, Design and Evaluation of Man-Machine Systems. Oulu, Finland.

Stahre J. and L. Mårtensson (1992). Supervising the CIM system - new roles for the operator. Proceedings of the Industrial Automation'92 Conference. Singapore, 199-211.

Sylla C. (1992). Modelling the pairing of human and machine-vision in industrial inspection tasks. Proceedings of the 7th IFAC/IFIP/IFORS/IMACS/ISPE Symposium on Information Control Problems in Manufacturing Technology, INCOM'92. Toronto, Canada, 33-8.

\section{BIOGRAPHY}

Anders Adlemo received his M.Sc. in Electrical Engineering at Lund University of Technology, Lund, Sweden, in 1981, and his Ph. D in Computer Engineering at Chalmers University of Technology, Göteborg, Sweden, in 1993.

Dr. Adlemo is currently a researcher at the Department of Computer Engineering at Chalmers University of Technology. His current research interests include distributed computing systems, fault tolerant systems, and complex flexible manufacturing systems.

Sven-Arne Andréasson received his B.A. in Physics and Mathematics at the University of Göteborg, Sweden, in 1971, M.Sc. in Electrical Engineering at Chalmers University of Technology, Göteborg, Sweden, in 1976, and Ph. D. in Computer Science at Chalmers University of Technology, Göteborg, Sweden, in 1986.

Dr. Andréasson is currently an associate professor at the Department of Computing Science at Chalmers University of Technology. His current research interests include distributed computing systems, fault-tolerant systems, databases and complex flexible manufacturing systems.

Dr. Andréasson is a member of the Association for Computing Machinery (ACM), and the IEEE Computer Society. 